Tropical Journal of Pharmaceutical Research November 2021; 20 (11): 2401-2411

ISSN: $1596-5996$ (print); 1596-9827 (electronic) (C) Pharmacotherapy Group, Faculty of Pharmacy, University of Benin, Benin City, 300001 Nigeria

\title{
Clinical efficacy and safety of Kanglaite injection, adjuvant cemcitabine and cisplatin chemotherapy for advanced non-small-cell lung cancer: A systematic review and meta- analysis
}

\author{
Yumei Dong ${ }^{1}$, Shihong Wei ${ }^{1}$, Xiaojun $\mathrm{Xia}^{2}$, Yuexiao $\mathbf{Q i}^{1}$, Haixia Song${ }^{1}$, Yaqin \\ Cai ${ }^{1}$, Liyun Guo ${ }^{1 *}$ \\ ${ }^{1}$ Department of Radiotherapy, ${ }^{2}$ Department of Hematology, Gansu Provincial Cancer Hospital, Lanzhou 730050, China
}

*For correspondence: Email: lifang20101@126.com; Tel: +86-015101312076

Sent for review: 7 July 2021

Revised accepted: 11 October 2021

\begin{abstract}
Purpose: To investigate the effectiveness and safety of the combination of Kanglaite injection (KLTi) and gemcitabine and cisplatin (GP) chemotherapy in the treatment of advanced non-small cell lung cancer (NSCLC).

Methods: PubMed, Web of Science, Embase, Cochrane Library, CNKI, Wan-Fang, CBM, and CQVIP were comprehensively searched from January 2010 till November 2020. Randomized controlled trials (RCTS) of KLTi plus GP in the treatment of NSCLC were selected and assessed for inclusion. Review Manager 5.3 software was used for meta-analysis.

Results: Twenty-five RCTs on advanced NSCLC examined the inclusion criteria. The meta-analysis showed that compared with GP chemotherapy alone, KLTi plus GP chemotherapy significantly improved objective response rate (ORR) $(R R=1.36,95 \% \mathrm{Cl} 1.23-1.51, p<0.00001)$, disease control rate $(D C R)(R R=1.17,95 \% \mathrm{Cl} 1.11-1.23, p<0.00001)$, and reduced adverse drug reactions(ADRs) such as hair loss ( $R R=0.60,95 \% \mathrm{Cl} 0.47-0.76, p<0.0001)$, gastrointestinal reaction $(R R=0.68,95 \%$ $\mathrm{Cl} 0.62-0.75, p<0.00001)$, impairment of liver and kidney function $(R R=0.65,95 \% \mathrm{Cl} 0.53-0.80, p<$ 0.001 ), nervous system damage ( $R R=0.42,95 \% \mathrm{Cl} 0.26-0.69, p=0.0005)$, myelosuppression (I-II phase) $(R R=0.79,95 \% \mathrm{Cl} 0.66-0.95, p=0.01)$, myelosuppression (III-IV phase) (RR $=0.44,95 \%$ Cl0.27 - 0.72, $p=0.001)$, anemia $(R R=0.74,95 \% \mathrm{Cl} 0.60-0.91, p=0.006)$, leukopenia $(R R=0.78$, $95 \% \mathrm{Cl} 0.69,0.87, p<0.0001)$, thrombocytopenia $(R R=0.59,95 \% \mathrm{Cl} 0.49,0.72, p<0.00001)$, hypochromia $(R R=0.74,95 \% \mathrm{Cl} 0.59,0.92, p=0.008)$.

Conclusion: KLTi adjuvant GP chemotherapy reduces adverse effects in patients with advanced NSCLC. Thus, KLTi might be an effective and safe intervention for NSCLC.
\end{abstract}

Keywords: Lung cancer, Gemcitabine and Cisplatin chemotherapy, Kanglaite injection, Meta-analysis, Randomized controlled trial (RCT)

\begin{abstract}
This is an Open Access article that uses a funding model which does not charge readers or their institutions for access and distributed under the terms of the Creative Commons Attribution License (http://creativecommons.org/licenses/by/4.0) and the Budapest Open Access Initiative (http://www.budapestopenaccessinitiative.org/read), which permit unrestricted use, distribution, and reproduction in any medium, provided the original work is properly credited.
\end{abstract}

Tropical Journal of Pharmaceutical Research is indexed by Science Citation Index (SciSearch), Scopus, International Pharmaceutical Abstract, Chemical Abstracts, Embase, Index Copernicus, EBSCO, African Index Medicus, JournalSeek, Journal Citation Reports/Science Edition, Directory of Open Access Journals (DOAJ), African Journal Online, Bioline International, Open-J-Gate and Pharmacy Abstracts 


\section{INTRODUCTION}

At present, lung cancer is still one of the major diseases that threaten human health. In recent decades, the morbidity and mortality rates of lung cancer have witnessed a significant increase. In 2018, the International Agency for Research on Cancer reported that global new cases of cancer reached 18.1 million, out of which 9.6 million are reported to have died. Irrespective of gender, lung cancer is the most commonly diagnosed cancer $(11.6 \%$ of total cases) and the leading cause of cancer death $(18.4 \%$ of the total cancer deaths) [1]. However, in 2020, 19.3 million cases were diagnosed new cases of cancer, with almost 10.0 million deaths according to the International Agency for Research on Cancer. Female breast cancer has surpassed lung cancer for the first time as the most commonly diagnosed cancer. Lung cancer however, is still the leading cause of cancer-related death, with an estimated 1.8 million deaths (18\%). In China, there were 4.57 million new cancer cases in 2020 , with lung cancer cases at 0.82 million. In addition, the incidence of lung cancer still ranks first: the number of cancer deaths is 3 million, of which lung cancer is as high as 0.71 million [2].

Chemotherapy is one of the main treatment methods for advanced NSCLC. The GP regimen is considered to be the main regimen used to treat advanced NSCLC, in which cisplatin destroys DNA function, blocks its replication, and plays an anti-cancer effect by linking with DNA strands. Gemcitabine has also a certain effect on DNA, which could repair inhibition and enhance the anti-cancer effect by combining with cisplatin [3]. However, there are many adverse reactions to GP chemotherapy. Where patients have a poor record of physical fitness and poor chemotherapy tolerance, chemotherapy poses the risk of exacerbating their suffering, thus affecting the quality of their lives. It is therefore necessary to find an effective drug that can mitigate the effects of chemotherapy on patients with advanced non-small-cell lung cancer, and improve their quality of life.

Kanglaite injection (KLTi) is extracted from the traditional Chinese medicine Coix seed, which has the dual effects of anti-tumor and immune function regulation. Studies have shown that $\mathrm{KLTi}$ not only enhances the effects of chemotherapy for NSCLC and reduces adverse reactions, it also improves the immune function of patients $[4,5]$. Therefore, this study aims to conduct a comprehensive meta-analysis of the results of clinical randomized controlled trials (RCTs) on KLTi plus chemotherapy, as well as systematically evaluate the efficacy and safety of
KLTi adjuvant chemotherapy for advanced NSCLC.

\section{METHODS}

This meta-analysis was strictly performed following the Preferred Reporting Items for Systematic Reviews and Meta-Analyses guidelines [6]. Ethical approval was not necessary since meta-analysis was based on secondary data, and did not involve any individual patient.

\section{Eligibility criteria}

These studies were evaluated according to the following inclusion criteria: (1) locally advanced or metastatic (stages III - IV) NSCLC confirmed histologically or cytologically without surgical operations and radiotherapy to the thoracic primary lesion, as well as other traditional Chinese medicine treatment; (2) studies on randomized controlled trials (RCTs); (3) Patients in the experimental group received $\mathrm{KLTi}$ and GP combination chemotherapy, and patients in the control group received corresponding conventional GP chemotherapy; (4) The primary outcomes, i.e., primary tumor response rate (CR, PR, SD, PD, etc.) and toxicity, were reported. It excluded retrospective clinical trials, case reports, meeting abstracts, cohort studies, in vitro and animal studies, duplicated studies, reviews, letters, commentaries, and errata, and studies on patients that received surgery, radiotherapy, or other traditional Chinese medicine treatment during the intervention period were excluded.

\section{Literature sources and search strategies}

PubMed, Web of Science, Embase, Cochrane Library, CNKI, Wan-Fang, CBM, and CQVIP, were comprehensively searched from January 2010 to November. The following search terms were used: ("Kanglaite" [Mesh terms]) AND ("lung cancer" [Mesh terms] OR "lung Neoplasms" [Mesh terms]). Languages were limited to English and Chinese. The search results were downloaded for further evaluation.

\section{Study selection}

All studies that were utilized for this study were imported into EndNote reference management software. Two reviewers (Dong and Guo) independently assessed the titles and abstracts in strict accordance with the inclusion and exclusion criteria. A third senior investigator (Wei) resolved any disagreements between the 2 reviewers.

Trop J Pharm Res, November 2021; 20(11): 2402 


\section{Definition of outcome measures}

Clinical efficacy was evaluated in accordance with the Response Evaluation Criteria in Solid Tumors (RECIST), developed by World Health Organization (WHO) curative effect evaluation criteria. Enrolled patients were divided into 4 categories: complete response (CR), partial response $(P R)$, stable disease (SD), and progressive disease (PD). Objective response rate $(\mathrm{ORR})$ was considered as the primary endpoint, and ORR $=[(\mathrm{CR}+\mathrm{PR}) /$ total $] \times 100 \%$. Moreover, the disease control rate was defined as the objective response, that is DCR, and DCR $=[(\mathrm{CR}+\mathrm{PR}+\mathrm{SD}) /$ total $] \times 100 \%$. Adverse events or adverse drug reactions (ADEs or ADRs) were classified into 0 IV levels, according to the National Cancer Institute Common Toxicity Criteria version 4.0 (CTC4.0). Adverse events include toxic reactions and adverse reactions that occur before and after treatment.

\section{Data extraction}

In each included study, the following basic information was independently obtained by two assessors: the first author's name, published year, study design and methodology, sample size, age, gender, TNM stage, and histologically type, including squamous carcinoma (SQC), adenocarcinomas, adenosquamous carcinoma (ADC), and large-cell carcinomas, and the main outcome indicators, including ORR, DCR, and ADRs.

\section{Assessment of risk of bias (quality)}

Risk of bias assessment for all studies was performed by the two assessors, according to the Cochrane risk of bias assessment. Quality assessment of each trial was evaluated by the Review Manager 5.3 according to the Cochrane Handbook for Systematic Reviews of Interventions.[6]

\section{Statistical analysis}

Statistical analysis was performed using Review Manager 5.3 software. Risk ratios (RRs) with 95 $\%$ confidence interval $(95 \% \mathrm{Cl})$ were calculated for dichotomous variables. Heterogeneity was evaluated according to the magnitude of $p$-value and $\mathrm{I}^{2}$ index. Significant heterogeneity was present when $\mathrm{I}^{2}>50 \%$, while non-significant heterogeneity was considered when $p>0.05$ and $\mathrm{I}^{2} \leq 50 \%$. Thus, a fixed-effect model was adopted when heterogeneity was minor. Subgroup or sensitivity analysis was used to explore heterogeneity where necessary. Funnel plots were used to assess publication bias.

\section{RESULTS}

\section{Selected literature}

Following the PRISMA, Figure 1 flow diagram illustrated the selection process of eligible studies. Databases were retrieved and 519 records were identified based on the established search strategy. The 294 duplicate records were excluded after their titles and abstracts were screened. Furthermore, full-text articles of 79 publications were assessed for eligibility, of which 54 were excluded because they did not report ADEs, reviews, or mechanistic articles, and the study content was inconsistent. Finally, 25 reports were included in the meta-analysis, and 2194 patients (experimental groups: 1100 cases; control groups: 1094 cases) who met the inclusion criteria were included, and data was extracted from these trials and used for the qualitative analysis.

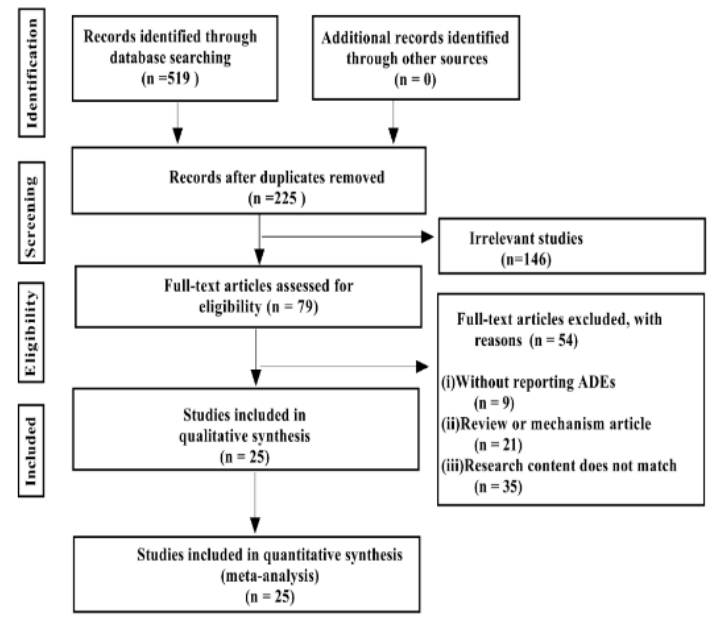

Figure 1: Flow chart for study selection

\section{Characteristics of included studies}

Table 1, Table 2, Table 3, Table 4 and Table 5 showed the characteristics of the included studies. All the selected literature had been published in China between 2010 and 2020 with no significant baseline differences. All the studies were RCTs. The age range was 27 - 78 years, with males and females totaling 1386 and 878 in number respectively (Table 1 ). The main types of the pathology of advanced NSCLC consisted of squamous cell carcinoma, adenocarcinomas, adenosquamous carcinoma, and large cell carcinomas (Table 2). Twenty-five studies were phase III-IV phase studies, and they all reported outcomes of clinical efficacy and adverse reactions. Twenty-two studies reported $\mathrm{KLTi}$ was administered intravenously at $200 \mathrm{~mL} /$ time, 1 to 3 
week/cycle, and 1 to 4 cycles (Table 3 ). All adverse reactions were reported in the trials, including myelosuppression, hair loss, gastrointestinal reaction, impairment of liver and kidney function and nervous system damage, of which anemia, leukopenia, thrombocytopenia, decreased hemoglobin, were observed in myelosuppression (Table 4 and Table 5).

\section{Risk of bias in included trials}

According to the Cochrane risk of bias estimation, the risk of bias was evaluated for each study included in the trial. All included studies mentioned "random", of which only ten studies $[9,11,14,18,23,24,26-29]$ definitely referred to random number table, and one trial [8] with random drawing. No trial reported information on allocation concealment, blinding of participants and personnel, or blinding of outcome assessment (Figure 2).

\section{Clinical efficacy analysis}

All 25 trials [3,5,7-29] reported the efficacy of tumor responses in detail (Figures 3 and 4 ). The
ORR and DCR were used to assess the shortterm efficacy, according to the RECIST criteria. There was no statistical heterogeneity among the trials in the ORR $\left(p=0.94, \mathrm{I}^{2}=0 \%\right)$ and DCR $(p$ $\left.=0.71, \mathrm{I}^{2}=0 \%\right)$, respectively. Therefore, the fixed effect model was applied in this pooled analysis. Our results indicated that $\mathrm{KLTi}$ combined with GP chemotherapy was superior to GP chemotherapy in terms of ORR (RR $=1.36$, $95 \% \mathrm{Cl} 1.23-1.51, p<0.00001))$ and DCR (RR $=1.16,95 \% \mathrm{Cl} 1.11-1.23, p<0.00001)$.

\section{Subgroup analysis}

The subgroup analysis was performed in order to examine the effect of different doses or cycles KLTi united GP regimens on ORR and DCR. The doses of $\mathrm{KLTi}$ were $100 \mathrm{~mL} /$ day in two trials [14,23], and in another trial it was $60 \mathrm{ml} /$ day [7]. In the other 22 studies [5,8-13,15-22,24-29], the dose of $\mathrm{KLTi}$ was $200 \mathrm{~mL} /$ day. In the subgroup analysis, it was found that irrespective of the dosage of KLTi that was combined with GP, the ORR and DCR (Figures 5 and 6) could be improved.

Table 1: Characteristics (gender and age) of the included studies

\begin{tabular}{|c|c|c|c|}
\hline $\begin{array}{l}\text { Total sample } \\
(\mathrm{T}(\mathrm{E} / \mathrm{C}))\end{array}$ & Gender (M/F) & Age (years) (range, mean) & Ref no. \\
\hline 98(49/49) & $52 / 46$ & $27 \sim 74$ & [7] \\
\hline $60(30 / 30)$ & $E(16 / 14) ; C(15 / 15)$ & $E(60.46 \pm 11.17) ; C(59.53 \pm 11.68)$ & [8] \\
\hline $102(51 / 51)$ & $E(29 / 22) ; C(30 / 21)$ & $\mathrm{E}(62.8 \pm 4.2) ; \mathrm{C}(62.7 \pm 4.5)$ & [9] \\
\hline $82(41 / 41)$ & $E(25 / 16) ; C(29 / 12)$ & $\mathrm{E}(55.13 \pm 4.85) ; C(54.51 \pm 5.93)$ & [10] \\
\hline $120(60 / 60)$ & $E(69 / 51) ; C(34 / 27)$ & $E(55.69 \pm 5.86)) ; C(55.75 \pm 5.90)$ & [11] \\
\hline $200(99 / 101)$ & $E(29 / 37) ; C(65 / 36)$ & $E(60.58 \pm 6.13) ; C(61.02 \pm 6.25)$ & [3] \\
\hline $70(35 / 350$ & $E(16 / 14) ; C(18 / 12)$ & $\mathrm{E}(59 \sim 78) ; \mathrm{C}(60 \sim 77)$ & [12] \\
\hline $62(31 / 31)$ & $E(13 / 12) ; C(16 / 11)$ & $E(61.13 \pm 3.1) ; C(63.02 \pm 3.5)$ & [13] \\
\hline $48(25 / 23)$ & $E(18 / 7) ; C(19 / 4)$ & $\mathrm{E}(60 \sim 75) ; C(60 \sim 73)$ & [14] \\
\hline $72(38 / 35)$ & $E(21 / 14) ; C(23 / 12)$ & $E($ mean age 64$) ; C($ mean age 64.8$)$ & [15] \\
\hline $82(42 / 40)$ & $E(27 / 15) ; C(25 / 15)$ & $E(61.16 \pm 1.2) ; C(59.53 \pm 1.39)$ & [16] \\
\hline $124(62 / 62)$ & $E(29 / 14) ; C(26 / 18)$ & $\mathrm{E}(47.09 \pm 4.02) ; \mathrm{C}(47.12 \pm 4.08)$ & {$[17]$} \\
\hline $68(34 / 34)$ & $E(42 / 21) ; C(39 / 24)$ & $E(56.63 \pm 5.17) ; C(56.91 \pm 6.33)$ & [18] \\
\hline $78(39 / 39)$ & $E(25 / 14) ; C(27 / 12)$ & $\mathrm{E}(68.25 \pm 6.68) ; \mathrm{C}(67.50 \pm 7.12)$ & [19] \\
\hline $86(43 / 43)$ & $E(29 / 14) ; C(29 / 14)$ & $E(60.07 \pm 5.43) ; C(59.79 \pm 6.02)$ & [20] \\
\hline $80(40 / 40)$ & $E(25 / 15) ; C(26 / 14)$ & $E(58.9 \pm 5.7) ; C(57.5 \pm 5.4)$ & [21] \\
\hline 78(39/39) & $E(29 / 10) ; C(26 / 13)$ & $\mathrm{E}(64.3 \pm 7.5) ; \mathrm{C}(63.5 \pm 8.9)$ & [22] \\
\hline $115(58 / 57)$ & $E(32 / 26) ; C(57 / 33)$ & $E(52.1 \pm 3.5) ; C(51.9 \pm 3.8)$ & [23] \\
\hline $98(49 / 49)$ & $E(32 / 17) ; C(31 / 18)$ & $E(62.5 \pm 5.8) ; C(62.8 \pm 4.5)$ & [24] \\
\hline 69(36/33) & $E(20 / 13) ; C(21 / 15)$ & $E(68.12 \pm 8.90) ; C(66.82 \pm 7.21)$ & [25] \\
\hline 137(70/67) & $E(41 / 29) ; C(37 / 30)$ & $E(64.8 \pm 7.2) ; C(63.4 \pm 7.0)$ & [5] \\
\hline $80(40 / 40)$ & $E(26 / 14) ; C(28 / 12)$ & $E(61.4 \pm 9.27) ; C(63.1 \pm 10.34)$ & [26] \\
\hline $47(24 / 23)$ & $E(18 / 6) ; C(17 / 7)$ & $\mathrm{E}(57.84 \pm 7.84) ; \mathrm{C}(56.98 \pm 7.97)$ & [27] \\
\hline $62(32 / 30)$ & $E(20 / 12) ; C(16 / 14)$ & $\mathrm{E}(65 \sim 72) ; \mathrm{C}(65 \sim 71)$ & [28] \\
\hline $85(44 / 42)$ & $E(25 / 18) ; C(24 / 18)$ & $E(60.22 \pm 3.24) ; C(57.12 \pm 3.58)$ & [29] \\
\hline
\end{tabular}

T: total; E: experiment group (Kanglaite injection plus GP chemotherapy); C: control group (GP chemotherapy); M: man; F: female 
Table 2: Characteristics (Pathological types and TNM stages) of the included studies

\begin{tabular}{|c|c|c|}
\hline Pathological type (SCC/A/others) & Stage of TNM (range, mean) & Ref no. \\
\hline$E(17 / 22 / 10) ; C(19 / 21 / 9)$ & (IIla24, IIIb34, IV40) & [7] \\
\hline NSCLC & $\mathrm{E}(\mathrm{IIIb} 27, \mathrm{IV} 3) ; \mathrm{C}(\mathrm{IIIb} 25, \mathrm{IV} 5)$ & [8] \\
\hline$E(14 / 29 / 8) ; C(14 / 29 / 8)$ & E(IIIa14,IIIb17, IV20);C(IIla13,IIIb16, IV22) & [9] \\
\hline NSCLC & (advanced) & {$[10]$} \\
\hline$E(12 / 36 / 12) ; C(13 / 38 / 10)$ & E(IIla12, IIlb39, IV9);C(IIla12, IIIb38, IV11) & [11] \\
\hline$E(44 / 52 / 3) ; C(43 / 55 / 3)$ & $\mathrm{E}(\mathrm{III} 48, \mathrm{IV} 51) ; \mathrm{C}(\mathrm{III} 50, \mathrm{IV} 51)$ & [3] \\
\hline$E(19 / 12 / 4) ; C(17 / 15 / 3)$ & $\mathrm{E}(\mathrm{III} 12, \mathrm{IV} 23) ; \mathrm{C}(\mathrm{III} 10, \mathrm{IV} 25)$ & {$[12]$} \\
\hline NSCLC & (advanced) & [13] \\
\hline $\mathrm{E}(7 / 18) ; C(9 / 14)$ & $\mathrm{E}(\mathrm{IIIb} 10, \mathrm{IV} 15 ; \mathrm{C}(\mathrm{IIIb} 8, \mathrm{IV} 15)$ & {$[14]$} \\
\hline $\mathrm{E}(22 / 12 / 1) ; \mathrm{C}(19 / 15 / 1)$ & $\mathrm{E}(\mathrm{III} 12, \mathrm{IV} 23) ; \mathrm{C}(\mathrm{III} 10, \mathrm{IV} 25)$ & {$[15]$} \\
\hline $\mathrm{E}(22 / 19 / 2) ; \mathrm{C}(20 / 22 / 1)$ & $\mathrm{E}(\mathrm{IIIb} 23,20) ; \mathrm{C}(\mathrm{III} \mathrm{lb} 25, \mathrm{IV} 18)$ & {$[16]$} \\
\hline $\mathrm{E}(8 / 20 / 14) ; C(7 / 19 / 14)$ & E(IIIb15,IV27);C(IIIB13,IV27) & [17] \\
\hline$E(21 / 13) ; C(23 / 11)$ & NA & [18] \\
\hline$E(16 / 17 / 6) ; C(17 / 19 / 3)$ & (advanced) & [19] \\
\hline$E(24 / 18 / 1) ; C(23 / 18 / 2)$ & E(III34, IV9);C(III33, IV10) & {$[20]$} \\
\hline$E(17 / 11 / 12) ; C(15 / 10 / 15)$ & $\mathrm{E}(\mathrm{III} 27, \mathrm{IV} 13) ; \mathrm{C}(\mathrm{III} 29, \mathrm{IV} 11)$ & [21] \\
\hline$E(20 / 12 / 7) ; C(21 / 10 / 8)$ & $\mathrm{E}(\mathrm{III} 25, \mathrm{IV} 14) ; \mathrm{C}(\mathrm{III} 27, \mathrm{IV} 12)$ & {$[22]$} \\
\hline NSCLC & $\mathrm{E}(\mathrm{III} 32, \mathrm{IV} 26) ; \mathrm{C}(\mathrm{III} 57, \mathrm{IV} 33)$ & [23] \\
\hline$E(23 / 26) ; C(24 / 25)$ & $\mathrm{E}(\mathrm{IIla} 21, \mathrm{IIlb} 24, \mathrm{IV} 4) ; \mathrm{C}(\mathrm{IIla} 20, \mathrm{Illb} 25, \mathrm{IV} 4)$ & {$[24]$} \\
\hline$E(16 / 15 / 2) ; C(16 / 17 / 3)$ & (advanced) & {$[25]$} \\
\hline $\mathrm{E}(34 / 31 / 5) ; \mathrm{C}(32 / 31 / 4)$ & $\mathrm{E}(\mathrm{IIIb} 45$, IV25);C(IIIb41, IV26) & [5] \\
\hline$E(18 / 20 / 2) ; C(17 / 20 / 3)$ & $\mathrm{E}(\mathrm{III} 22, \mathrm{IV} 18) ; \mathrm{C}(\mathrm{III} 21, \mathrm{IV} 19)$ & {$[26]$} \\
\hline $\mathrm{E}(6 / 15 / 3) ; \mathrm{C}(7 / 13 / 4)$ & E(IIla5, IIIb14, IV5);C(IIla5, IIIb13, IV5) & {$[27]$} \\
\hline$E(16 / 14 / 2) ; C(15 / 13 / 2)$ & $\mathrm{E}(\mathrm{IIIb} 17, \mathrm{IV} 15) ; \mathrm{C}(\mathrm{IIIb} 17, \mathrm{IV} 13)$ & [28] \\
\hline$(38 / 45 / 2)$ & $($ III41, IV44) & [29] \\
\hline
\end{tabular}

T: total; E: experiment group (Kanglaite injection plus GP chemotherapy); C: control group (GP chemotherapy); SCC: squamous cell carcinoma, A: adenocarcinomas; TNM: tumor node metastasis.

Table 3: Outcomes of included meta-analyses on KLTi in combination with chemotherapy for advanced NSCLC

\begin{tabular}{lcc}
\hline & Outcome & Ref no. \\
\hline ORR((CR+PR)/TS) & DCR(CR+PR+SD)/TS) & {$[7]$} \\
\hline$E(20 / 49) ; C(15 / 49)$ & $E(34 / 49) ; C(29 / 49)$ & {$[8]$} \\
$E(16 / 30) ; C(11 / 30)$ & $E(24 / 30) ; C(20 / 30)$ & {$[9]$} \\
$E(22 / 51) ; C(18 / 51)$ & $E(39 / 51) ; C(37 / 51)$ & {$[10]$} \\
$E(25 / 41) ; C(17 / 41)$ & $E(36 / 41) ; C(32 / 41)$ & {$[11]$} \\
$E(38 / 60) ; C(29 / 60)$ & $E(51 / 60) ; C(43 / 60)$ & {$[3]$} \\
$E(43 / 99) ; C(34 / 101)$ & $E(82 / 99) ; C(70 / 101)$ & {$[12]$} \\
$E(19 / 35) ; C(13 / 35)$ & $E(28 / 35) ; C(27 / 35)$ & {$[13]$} \\
$E(25 / 31) ; C(13 / 31)$ & $E(29 / 31) ; C(23 / 31)$ & {$[14]$} \\
$E(10 / 25) ; C(8 / 23)$ & $E(18 / 25) ; C(15 / 23)$ & {$[15]$} \\
$E(15 / 38) ; C(12 / 35)$ & $E(28 / 38) ; C(26 / 35)$ & {$[16]$} \\
$E(18 / 42) ; C(14 / 40)$ & $E(29 / 42) ; C(26 / 40)$ & {$[18]$} \\
$E(17 / 62) ; C(15 / 62)$ & $E(36 / 62) ; C(28 / 62)$ & {$[19]$} \\
$E(29 / 34) ; C(21 / 34)$ & $E(29 / 34) ; C(21 / 34)$ & {$[21]$} \\
$E(12 / 39) ; C(8 / 39)$ & $E(29 / 39) ; C(24 / 39)$ & {$[22]$} \\
$E(19 / 43) ; C(12 / 43)$ & $E(37 / 43) ; C(28 / 43)$ & {$[23]$} \\
$E(26 / 40) ; C(12 / 40)$ & $E(34 / 40) ; C(28 / 40)$ & {$[24]$} \\
$E(20 / 39) ; C(15 / 39)$ & $E(32 / 39) ; C(28 / 39)$ & {$[5]$} \\
$E(27 / 48) ; C(16 / 57)$ & $E(46 / 48) ; C(37 / 57)$ & {$[26]$} \\
$E(23 / 49) ; C(21 / 49)$ & $E(39 / 49) ; C(38 / 49)$ & {$[27]$} \\
$E(9 / 36) ; C(8 / 33)$ & $E(22 / 36) ; C(22 / 33)$ & {$[28]$} \\
$E(19 / 70) ; C(15 / 67)$ & $E(47 / 70) ; C(42 / 67)$ & {$[29]$} \\
$E(22 / 40) ; C(21 / 40)$ & $E(36 / 40) ; C(35 / 40)$ & $E$ \\
$E(14 / 24) ; C(7 / 23)$ & $E(20 / 24) ; C(13 / 23)$ & \\
$E(11 / 32) ; C(10 / 30)$ & $E(19 / 32) ; C(14 / 30)$ & \\
$E(19 / 43) ; C(14 / 42)$ & $E(35 / 43) ; C(29 / 42)$ & \\
\hline
\end{tabular}

ORR: objective response rate; DCR: disease control rate; CR: complete remission; PR: partial remission; SD: stable disease; T: total sample; E: experiment group (Kanglaite injection plus GP chemotherapy); C: control group (GP chemotherapy). 
Table 4: ADEs of included meta-analyses on KLTi in combination with chemotherapy for advanced NSCLC

\begin{tabular}{|c|c|c|c|c|c|c|}
\hline & & ADES & & & & \\
\hline Hair loss (E/C) & Gastrointestinal & impairment of liver & Nervous system & Myelosu & ion $(E / C)$ & \\
\hline & reaction $(E / C)$ & $\begin{array}{l}\text { and Kidney's } \\
\text { function(E/C) }\end{array}$ & damage $(E / C)$ & $(I-I I)$ & (III-IV) & Ret no. \\
\hline$(15 / 34)$ & $(34 / 36)$ & $(16 / 22)$ & $(5 / 17)$ & $16 / 26$ & $/ 1 / 3$ & [7] \\
\hline- & $(14 / 15)$ & $(4 / 11)$ & - & - & - & [8] \\
\hline - & $(20 / 28)$ & $(5 / 11)$ & $(3 / 5)$ & - & - & [9] \\
\hline- & $(2 / 3)$ & $(0 / 3)$ & - & - & - & [10] \\
\hline- & $(16 / 25)$ & $(18 / 24)$ & - & - & - & [11] \\
\hline - & $(29 / 58)$ & $(7 / 9)$ & - & - & - & [3] \\
\hline - & $(14 / 26)$ & - & - & - & - & [12] \\
\hline - & $(1 / 2)$ & $(1 / 4)$ & - & - & - & [13] \\
\hline - & $(5 / 7)$ & $(1 / 6)$ & - & - & - & [14] \\
\hline- & $(17 / 24)$ & $(11 / 18)$ & - & - & - & [15] \\
\hline- & $(14 / 19)$ & - & - & $13 / 15$ & $/ 2 / 9$ & [16] \\
\hline$(13 / 26)$ & $(27 / 29)$ & $(14 / 15)$ & $(5 / 14)$ & $14 / 25$ & - & [17] \\
\hline$(11 / 10)$ & $(7 / 10)$ & $(4 / 3)$ & $(3 / 2)$ & $4 / 5$ & - & [18] \\
\hline- & $(1 / 8)$ & - & & - & - & [19] \\
\hline- & $(5 / 10)$ & - & $(4 / 7)$ & $7 / 8$. & $0 / 2$ & [20] \\
\hline$(1 / 2)$ & $(2 / 4)$ & $(2 / 0)$ & $(0 / 3)$ & - & - & [21] \\
\hline (20/27) & $(27 / 28)$ & $(18 / 20)$ & $(28 / 11)$ & - & - & [22] \\
\hline- & $(12 / 22)$ & $(3 / 11)$ & - & - & - & [23] \\
\hline - & $(13 / 23)$ & $(5 / 6)$ & - & - & - & [24] \\
\hline - & $(1 / 3)$ & - & - & - & - & [25] \\
\hline - & $(55 / 60)$ & - & - & $47 / 43$ & $13 / 22$ & [5] \\
\hline- & $(12 / 32)$ & - & - & - & - & [26] \\
\hline$(1 / 3)$ & $(1 / 2)$ & $(1 / 3)$ & - & - & - & [27] \\
\hline - & $(4 / 7)$ & $(0 / 4)$ & - & 12/17. & $2 / 4$ & [28] \\
\hline - & $(13 / 24)$ & $(3 / 4)$ & - & - & - & [29] \\
\hline
\end{tabular}

ADEs: adverse drug reactions; E: experiment group (Kanglaite injection plus GP chemotherapy); C: control group (GP chemotherapy)

Table 5: Characteristics of myelosuppression for advanced NSCLC

\begin{tabular}{|c|c|c|c|c|}
\hline Anemia (E/C) & Leukopenia (E/C) & Thrombocytopenia(E/C) & Hemoglobin decreased(E/C) & Ref no. \\
\hline- & - & - & - & [7] \\
\hline- & - & - & - & [8] \\
\hline- & $15 / 18$ & - & - & [9] \\
\hline- & - & - & - & [10] \\
\hline $13 / 15$ & $.11 / 19$ & - & $.12 / 17$ & [11] \\
\hline- & $22 / 57$ & $9 / 26$ & - & [3] \\
\hline- & $16 / 26$ & - & - & [12] \\
\hline- & $/ 1 / 1$ & - & - & [13] \\
\hline- & $3 / 8$ & - & $/ 4 / 9$ & [14] \\
\hline- & $/ 3 / 23$ & $20 / 22$ & - & [15] \\
\hline- & - & - & - & [16] \\
\hline- & - & - & - & [17] \\
\hline- & - & - & - & [18] \\
\hline $1 / 9$. & - & $0 / 6$ & - & [19] \\
\hline- & 7/10. & $2 / 4$ & - & [20] \\
\hline- & $2 / 0$ & $0 / 6$ & - & [21] \\
\hline- & $30 / 34$ & $25 / 25$ & 28/29. & [22] \\
\hline $13 / 24$ & 9/19. & - & - & [23] \\
\hline- & 12/13. & - & - & [24] \\
\hline $0 / 2$ & 0/2 & $1 / 2$ & - & [25] \\
\hline $46 / 50$ & 60/65 & $41 / 52$ & - & {$[5]$} \\
\hline- & - & - & - & [26] \\
\hline- & - & - & - & [27] \\
\hline- & $14 / 21$ & $11 / 16$ & & [28] \\
\hline- & $24 / 24$ & $14 / 9$ & $18 / 28$ & [29] \\
\hline
\end{tabular}

E: experiment group (Kanglaite injection plus GP chemotherapy); C: control group (GP chemotherapy) 
A

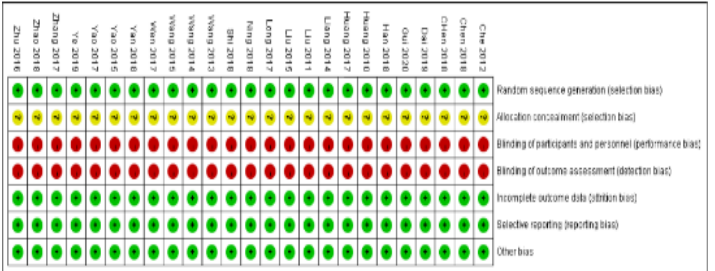

B

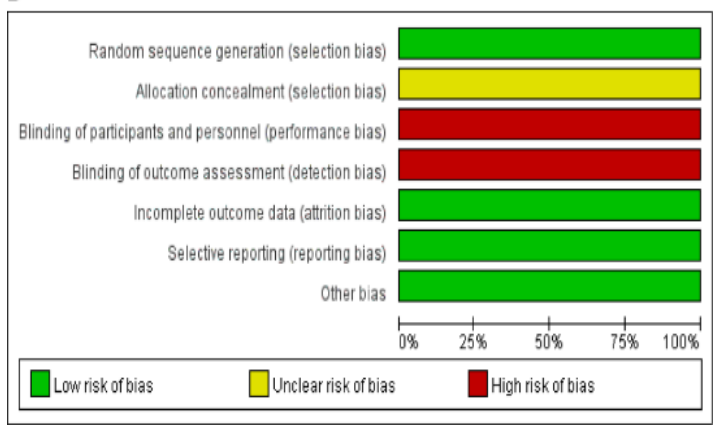

Figure 2: Risk of methodological bias. Risk of bias summary (A): review of authors' judgments about each risk of bias item for included studies. Risk of bias graph (B): review of authors' judgments about each risk of bias item presented as percentages across all included studies. Each color represents a different level of bias: red for high-risk, green for low-risk, and yellow for unclear-risk of bias

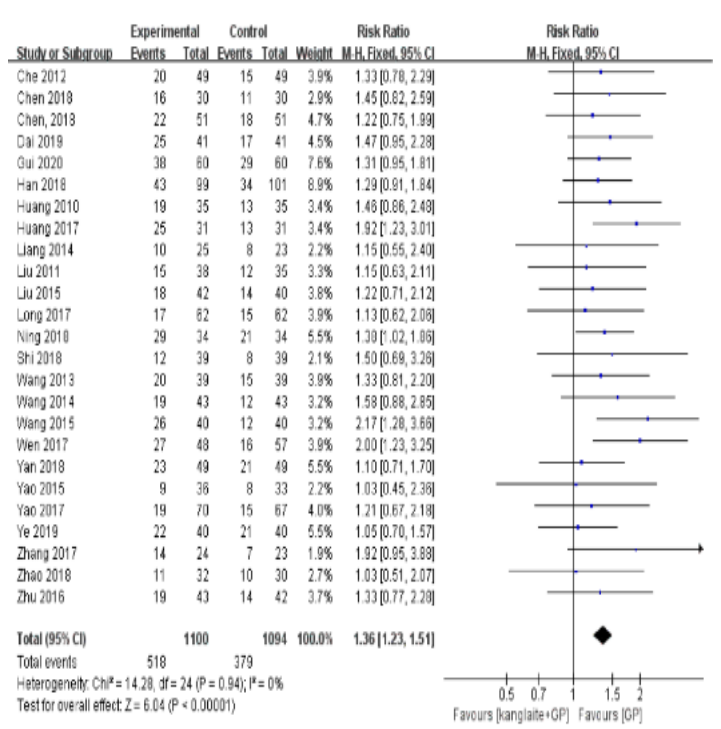

Figure 3: An analysis of objective response rate (ORR) between 2 groups. Forest plot of the comparison of ORR between the experimental and control group. Control group, GP chemotherapy alone group; experimental group, Kanglaite injection and GP chemotherapy combined group. The fixed-effects model was used.

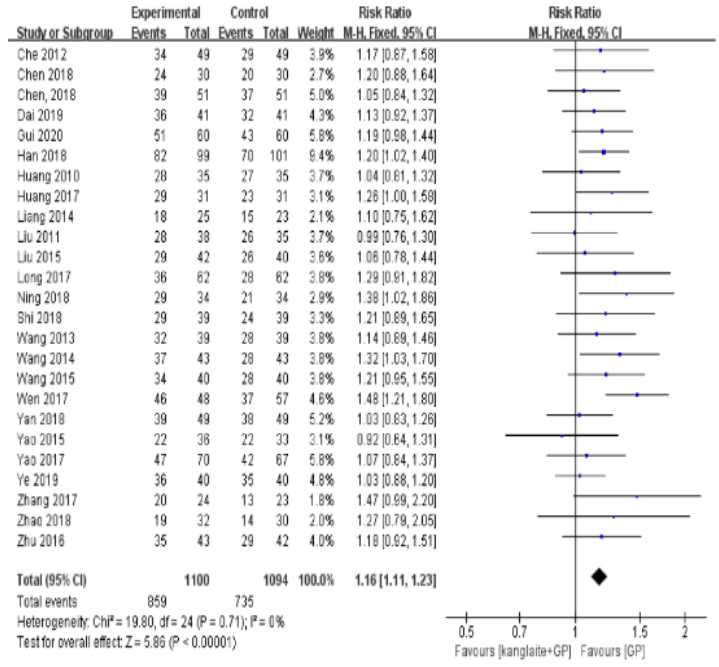

Figure 4: An analysis of disease control rate (DCR) between 2 groups. Forest plot of the comparison of DCR between the experimental and control group. Control group, GP chemotherapy alone group, experimental group, Kanglaite injection and GP chemotherapy combined group. The fixed-effects model was used

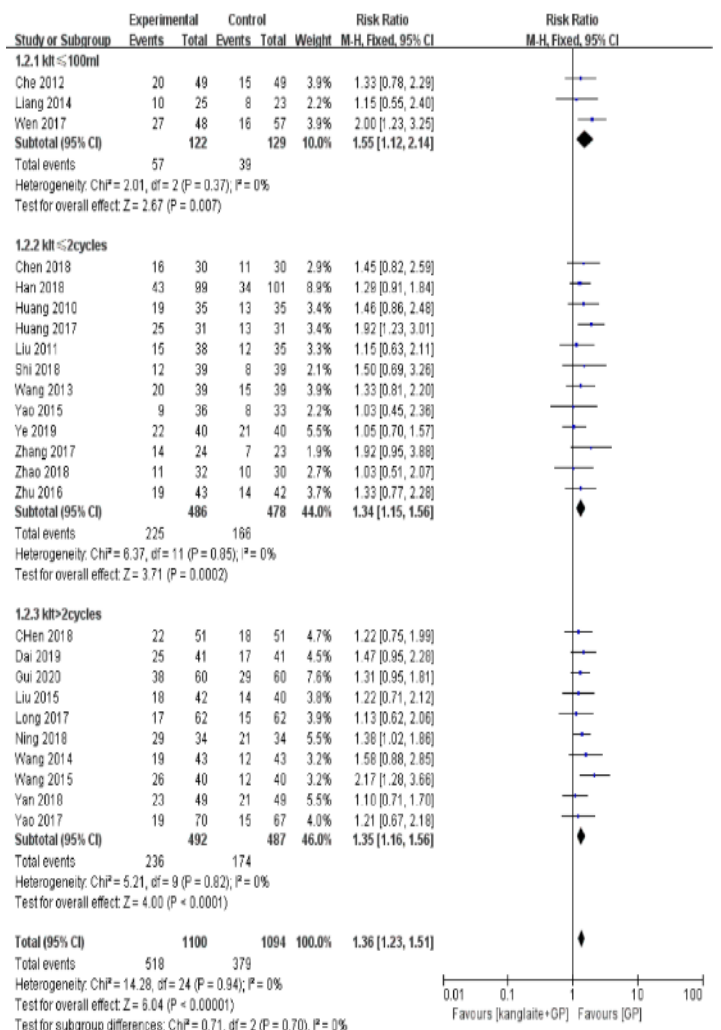

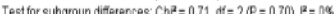

Figure 5: The subgroup analysis of objective response rate (ORR). 


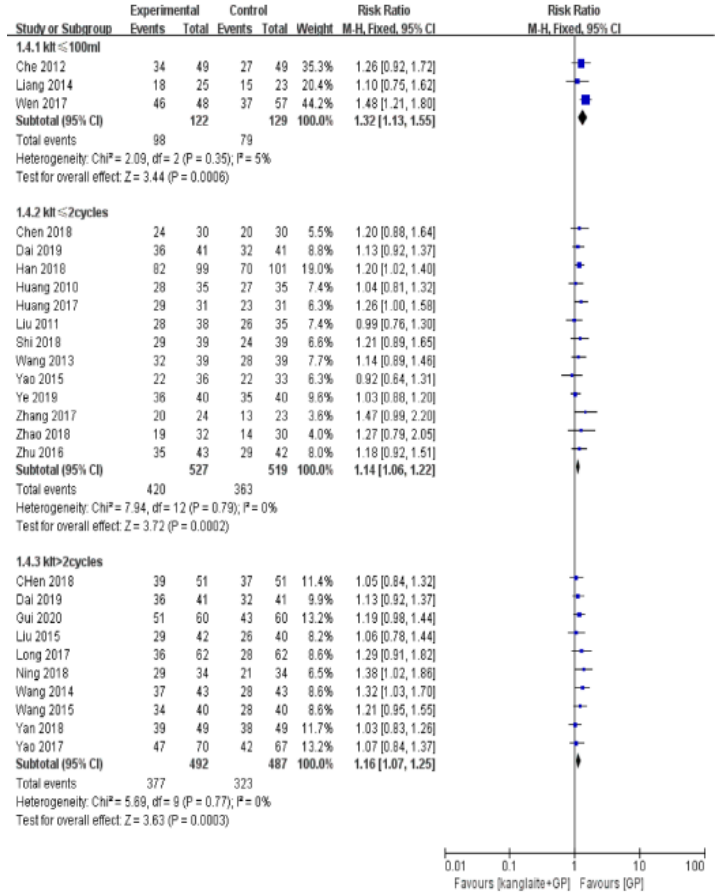

Figure 6: Subgroup analysis of disease control rate (DCR)

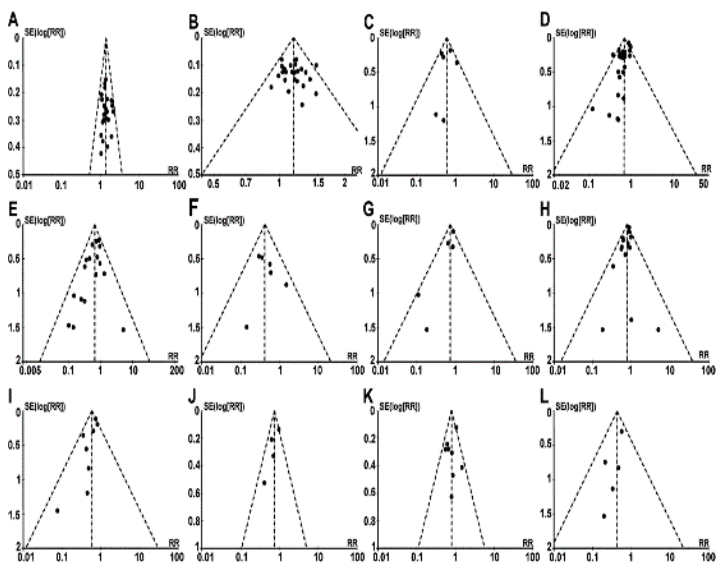

Figure 7: Publication bias analysis. Funnel plot of ORR (A), DCR (B), Hair loss (C), Gastrointestinal reaction (D), Impairment of liver and Kidney's function $(E)$, Nervous system damage (F), Anemia (G), Leukopenia $(\mathrm{H})$, Thrombocytopenia (I), Hemoglobin decreased (J), I-II phase myelosuppression (K), III-IV phase myelosuppression $(\mathrm{L})$

\section{ADRs}

A meta-analysis of the 25 included RCTs [3,5,729] showed ADRs of $K L T i$ in combination with GP chemotherapy for treating advanced NSCLC, including myelosuppression, hair loss, gastrointestinal reaction, impairment of liver and kidney function, and nervous system damage. Moreover, myelosuppression, anemia, leukopenia, thrombocytopenia, decreased haemoglobin were observed. It was found that the incidence of ADRs in the experiment group had a significantly lower level than the control group, except that there was no remarkably different incidence of I-II phase myelosuppression.

\section{Publication bias}

The publication bias was evaluated using the symmetrical funnel plots for ORR, DCR, the hair loss, gastrointestinal reaction, impairment of liver and kidney function, nervous system damage, anemia, leukopenia, thrombocytopenia, decreased hemoglobin, I-II phase myelosuppression, III-IV phase myelosuppression (Figure 7).

\section{DISCUSSION}

The findings of this meta-analysis indicate that combined $\mathrm{KLTi}$ and GP regimens can be an effective and safe method for the treatment of advanced NSCLC. This combination therapy model achieved favorable lung primary lesion response rates and superior survival rates with mild-to-moderate side effects. However, it should be noted that the quality of evidence supporting these findings is low.

Chemotherapy has generally been the firstchoice treatment option for advanced NSCLC. Gemcitabine and Cisplatin regimen is one of the effective treatment choices. Gemcitabine (2', 2'difluorodeoxycytidine) is a chemotherapeutic agent that inhibits DNA synthesis in dividing cells [30], and it is a third-generation cell cycle-specific anti-tumor drug with three anti-tumor mechanisms. In addition, Gemcitabine has relatively mild side effects, high safety, and low local irritation. Also, it is well tolerated by patients. At present, it is widely used in non-small cell lung cancer [3,5,7-29], pancreatic cancer [31], breast cancer [32], ovarian cancer [33], and bladder cancer [34], with good efficacy and safety.

Traditional Chinese medicine (TCM) injections are composed of active ingredients extracted from TCM or natural medicines using modern techniques and methods according to the theory and experience of TCM. KLTi is a novel antitumor Chinese medicine (TCMs) developed in China. It is a representative product of domestic Chinese medicine anti-tumor injections. Its main component is coix seed triglyceride, which has anti-tumor and immunity enhancement effects [35].

Kanglaite is an NF-kB inhibitor. In the study of four colorectal cancer cell lines HCT106, 
HCT116, LoVo, and CT26, it was found that tumor necrosis factor-alpha-mediating the activation of NF-kB, caused changes in epithelial-mesenchymal transition-related protein expression and increased migration and invasion in all four cell lines. However, these effects were inhibited by Kanglaite when used in combination with tumor necrosis factor-alpha. In a subcutaneous tumor model of CT26, tumor necrosis factor-alpha enhanced the tumorigenic ability of the cells, and again this was inhibited by Kanglaite. However, treatment with Kanglaite alone caused almost no inhibition of epithelialmesenchymal transition-mediated tumor growth, when cells were pretreated with tumor necrosis factor-alpha prior to injection. These results suggest that Kanglaite inhibits tumor necrosis factor-alpha-mediated epithelial-mesenchymal transition in colorectal cancer cell lines via the inhibition of NF-KB [36].

Another research demonstrated that Kanglaite markedly decreased the regulation of NF-kB/lkB expression and significantly increased the level of IL-2 and EGFR in C57BL/6 mice with Lewis lung carcinoma. Thus, $\mathrm{KLTi}$ has pronounced antitumor and immunostimulatory activities in C57BL/6 mice with Lewis lung carcinoma [37]. Clinically, kanglaite significantly reduces the expression of miRNA-21 in patients with advanced lung cancer, and provides objective evidence for the treatment of lung cancer [38]. In this comprehensive systematic review and metaanalysis, we comprehensively evaluated whether $\mathrm{KLTi}$ combined with GP chemotherapy versus GP chemotherapy alone would benefit patients with advanced NSCLC. Our results suggest that $\mathrm{KLTi}$ not only statistically improves clinical efficacy, it also reduces adverse reactions in advanced NSCLC.

In our meta-analysis, the clinical results were divided into objective response rate (ORR) and disease control rate (DCR). The results indicate that $\mathrm{KLTi}$ possesses superior clinical efficacy when combined with GP chemotherapy for the treatment of advanced NSCLC, and these results are consistent with previous studies [3,14]. Moreover, we found that $\mathrm{KLTi}$ combined with GP chemotherapy, reduced the incidence of adverse reactions in patients with advanced NSCLC. Appropriate drug selection is a major challenge in patients with advanced NSCLC, especially those who have complications from concomitant gastrointestinal system and circulatory diseases [35]. This study has certain limitations. First, the RCTs were uncommon in the use of $\mathrm{KLTi}$ in the treatment of advanced NSCLC. The descriptions of random sequence generation, allocation concealment, and blinding methods of the included clinical trials were not detailed, which might result in selected bias. As a result, there may been some overestimation or underestimation of the impact of $\mathrm{KLTi}$. Furthermore, the different stages of TNM and the pathological type diversity of the trials included in the study could form unbalanced baselines. Third, the use of different doses of $\mathrm{KLTi}$ in combination with chemotherapy and administration patterns may affect the efficacy and safety evaluation. Fourth, all included studies were published in Chinese, so the results of this systematic review may be affected by potential publication bias. In conclusion, for the clinical efficacy and safety of TCM, well-designed RCTs with large sample size and double-blind are needed for further assessment, so that Chinese TCM can be approved in international markets.

\section{CONCLUSION}

On the basis of the present meta-analysis, there is reasonable evidence that the combination of $\mathrm{KLTi}$ with chemotherapy may improve the ORR and survival outcomes in advanced NSCLC patients, as well as reduce the risk of hepatotoxicity, gastrointestinal reactions, neurotoxicity, and hepatotoxicity in patients with advanced NSCLC. However, a larger number of patients and prospective studies are required to fully establish the clinical efficacy and safety of this treatment.

\section{DECLARATIONS}

\section{Acknowledgement}

This study was supported by Gansu Provincial Healthcare Industry Science Research Project (no. GSWSKY-2015-35), Guiding Plan Project of Science and Technology Development in Lanzhou City, Gansu Province (no. 2019-ZD134), Gansu Provincial Administration of Traditional Chinese Medicine Research Project of Major Disease Prevention and Treatment of Traditional Chinese Medicine in 2018 (Project no. GZKZD-2018-03).

\section{Conflict of Interest}

No conflict of interest associated with this work.

\section{Contribution of Authors}

The authors declare that this work was done by the authors named in this article and all liabilities pertaining to claims relating to the content of this article will be borne by them.

Trop J Pharm Res, November 2021; 20(11): 2409 


\section{Open Access}

This is an Open Access article that uses a funding model which does not charge readers or their institutions for access and distributed under the terms of the Creative Commons Attribution License (http://creativecommons.org/licenses/by/ 4.0) and the Budapest Open Access Initiative (http://www.budapestopenaccessinitiative.org/rea d), which permit unrestricted use, distribution, and reproduction in any medium, provided the original work is properly credited.

\section{REFERENCES}

1. Bray F, Ferlay J, Soerjomataram I, Siegel RL, Torre LA, Jemal A. Global cancer statistics 2018: GLOBOCAN estimates of incidence and mortality worldwide for 36 cancers in 185 countries. CA Cancer J Clin 2018; 68(6): 394-424.

2. Erratum: Global cancer statistics 2018: GLOBOCAN estimates of incidence and mortality worldwide for 36 cancers in 185 countries. CA Cancer J Clin 2020; 70(4): 313.

3. Han B, Kang JY, Li FH. [J /CD]. Clinical analysis of Kanglaite Injection combined with GP chemotherapy in patients with advanced non-small cell lung cancer. Chinese Journal of Lung Diseases (Electronic Edition) 2018; 11(3): 304-308.

4. Hang M, Chen SB, Sun Q, et.al. Analysis of the efficacy and safety of GP regimen combined with Kanglaite injection in the treatment of advanced non-small cell lung cancer. Practical Journal of Cancer 2017; 32(2): 289-291.

5. Yao J, Song X. Clinical analysis of gemcitabine and cisplatin regimen combined with Kanglaite injection in the treatment of advanced non-small cell lung cancer. Journal of Clinical and Experimental Medicine 2017; 16(12): 1195-1198.

6. Moher D, Liberati A, Tetzlaff J, Altman DG. Preferred reporting items for systematic reviews and metaanalyses: the PRISMA statement. Plos Med 2009; 6(7): e1000097.

7. Che SH, Luo C, Liu Y, et al. Clinical Observation on the Therapeutic Effect of Kanglaite Injection plus GP on Advanced Non-Small Cell Lung Cancer. Anti-tumor Pharmacy 2012; 2(4): 286-289.

8. Chen C. Non-small Cell Lung Cancer Randomized Parallel Controlled Study of Advanced Kanglaite Injection combined with GP in Treatment. Journal of Practional Traditional Chinese Internal Medicine 2018; 32(3): 46-48.

9. Chen $Y$. The effect of GP regimen combined with Kanglaite in the treatment of advanced non-small cell lung cancer. Medical Journal of Chinese People's Health 2018; 30(8): 73-75.

10. Dai QG. Efficacy and safety analysis of Kanglaite injection combined with chemotherapy in the treatment of advanced non-small cell lung cancer. Family Medicine 2019; 3: 154.

11. Gui XM, Dai L, Yuan QW, et al. Effects of Kanglaite Injection on Levels of CD3+, CD4+, NK and CD4+ /CD8+ in Patients with Advanced Non-small Cell Lung Cancer. Chinese Archives of Traditional Chinese Medicine 2020; 38(5): 147-150.

12. Huang ZB, Zhu CY, Wu SX, et al. Effect of Kanglaite Combined with Chemical Therapy Advanced Non- small Cell Lung Cancer. China Pharmacy. 2010; 21(20): 18611860.

13. Huang ZC, Liu Y, Zeng CS, et al. Clinical efficacy and safety evaluation of Kanglaite combined with gemcitabine and cisplatin in the treatment of advanced non-small cell lung cancer. Contemporary Medicine 2017; 23(27): 71- 73.

14. Liang SM, Dong YH, Wang J, et al. Kanglaite Combined with GP in the Treatment of 25 cases of Senile Nonsmall-cell Lung Carcinoma. China Journal of Chinese Medicine 2014; 29(188): 11-12.

15. Liu JQ, Shang LQ, Li XC et al. Effect of kanglaite combined with chemical therapy advanced non-small cell lung cancer. Modern Oncology 2011; 19(10): 19741976.

16. Liu Y, Ma P. Therapeutic effect of Kanglaite injection combined with gemcitabine and cisplation on advanced non-small cell lung cancer. Chinese Journal of Practical Medicine 2015; 42(4):43-45.

17. Long SG, Xiao YL. The clinical efficacy Kanglaite combined with GP regimen in the treatment of advanced non-small cell lung cancer and its influence of various indicators of immune function. Anti Infect Pharm 2017; 14(2): 377-380.

18. Ning SF. Effect of Kanglaite Injection combined with chemotherapy in the treatment of non-small cell lung cancer and its influence on T lymphocyte subsets. China Modern Medicine 2018; 25(29): 18-21.

19. Shi EJ, Yi SP, Yuan CF. The clinical efficacy of Kanglaite combined with gemcitabine and cisplatin in the treatment of patients with advanced non-small cell lung cancer. Medical Equipment 2018; 31(20): 99-100.

20. Wang $L$. The effect of the immune function Kanglaite combined with chemotherapy in the treatment of patients with non-small cell lung cancer and its clinical efficacy analysis. Lab Med Clin 2014; 11(8): 1072-1075.

21. Wang L, Wang W, Atican K. The Curative Effect Observation of Kanglaite Injection Combining with Cisplatin in the Treatment of Lung Cancer. Practical Integrated Traditional Chinese and Western Medicine 2015; 15(6): 10-12.

22. Wang YY, Ye L, Liu K. Effects of Kanglaite Injection Combined with Chemotherapy in Treatment of Nonsmall-Cell Lung Cancer. Journal of Hubei Medical College 2013; 32(5): 387-390.

23. Wen $T$. The therapeutic effect of Kanglaite combined with GP regimen in the treatment of patients with advanced non-small cell lung cancer. Primary Medical Forum 2017; 21(10): 1225-1227.

Trop J Pharm Res, November 2021; 20(11): 2410 
24. Yan $Q H$, Xing GC, Pan Q. Observation of the effect of GP regimen combined with Kanglaite in the treatment of advanced non-small cell lung cancer. Practical combination of Chinese and Western medicine clinical 2018; 18(9): 112-121.

25. Yao DJ. Clinical efficacy and safety of Kanglaite combined with gemcitabine and cisplatinum chemotherapy in the treatment of advanced non - small cell lung cancer. Chin J Clin Pharmacol 2015; 31(15): 1494-1496.

26. Ye $C Y$, Zhao FC, Xing SS. Clinical Observation of Kanglaite Injection Combined with Gemcitabine Cisplatin Regimen for Advanced Non-small Cell Lung Cancer. Clinical Research 2019; 27(7): 24-26.

27. Zhang XF. Kanglaite adjuvant treatment of 47 cases of non-small cell lung cancer. Capital Food and Medicine 2017; 10: 79.

28. Zhao XG, Cheng $Y$, Shang $W$, et al. Effects of kanglaite injection combined with gemcitabine on advanced nonsmall cell lung cancer in the aged. Henan Medical Research 2018; 27(15): 2713-2715.

29. Zhu JJ, You YJ. Improvement Effect of Kanglaite Injection Combined with GP Program on the Immunity of Patients with Non-Small-Cell Lung Cancer at Advanced Stage. Henan Traditional Chinese Medicine 2016; 36(11): 1943-1945.

30. Messing EM, Tangen CM, Lerner SP, Sahasrabudhe DM, Koppie TM, Wood DJ, Mack PC, Svatek RS, Evans CP, Hafez KS, Culkin DJ, Brand TC, Karsh LI, Holzbeierlein $J M$, Wilson SS, Wu $G$, Plets $M$, Vogelzang $N J$, Thompson IJ. Effect of Intravesical Instillation of Gemcitabine vs Saline Immediately Following Resection of Suspected Low-Grade Non-Muscle-Invasive Bladder Cancer on Tumor Recurrence: SWOG 50337 Randomized Clinical Trial. JAMA 2018; 319(18): 18801888.

31. Motoi $F$, Kosuge $T$, Ueno $H$, Yamaue $H$, Satoi S, Sho $M$, Honda G, Matsumoto I, Wada K, Furuse J, Matsuyama $Y$, Unno M. Randomized phase II/III trial of neoadjuvant chemotherapy with gemcitabine and S-1 versus upfront surgery for resectable pancreatic cancer (Prep02/JSAP05). Jpn J Clin Oncol 2019; 49(2): 190-194.

32. Zhang J, Lin $Y$, Sun $X J$, Wang $B Y$, Wang $Z H$, Luo JF, Wang $L P$, Zhang S, Cao J, Tao ZH, et al. Biomarker assessment of the CBCSG006 trial: a randomized phase III trial of cisplatin plus gemcitabine compared with paclitaxel plus gemcitabine as first-line therapy for patients with metastatic triple-negative breast cancer. Ann Oncol 2018; 29(8): 1741-1747.

33. Berg T, Nottrup TJ, Roed H. Gemcitabine for recurrent ovarian cancer - a systematic review and meta-analysis. Gynecol Oncol 2019; 155(3): 530-537.

34. Coen JJ, Zhang P, Saylor PJ, Lee CT, Wu CL, Parker W, Lautenschlaeger T, Zietman AL, Efstathiou JA, Jani AB, et al. Bladder Preservation With Twice-a-Day Radiation Plus Fluorouracil/Cisplatin or Once Daily Radiation Plus Gemcitabine for Muscle-Invasive Bladder Cancer: NRG/RTOG 0712-A Randomized Phase II Trial. J Clin Oncol 2019; 37(1): 44-51.

35. Wen J, Yang $T$, Wang J, Ma X, Tong $Y$, Zhao $Y$. Kanglaite Injection Combined with Chemotherapy versus Chemotherapy Alone for the Improvement of Clinical Efficacy and Immune Function in Patients with Advanced Non-Small-Cell Lung Cancer: A Systematic Review and Meta-Analysis. Evid Based Complement Alternat Med 2020; 2020: 8586596.

36. Shi G, Zheng $X$, Zhang S, Wu X, Yu F, Wang Y, Xing F. Kanglaite inhibits EMT caused by TNF-alpha via NFkappaBeta inhibition in colorectal cancer cells. Oncotarget 2018; 9(6): 6771-6779.

37. Pan $P$, Wu Y, Guo ZY, Wang R, Wang YJ, Yuan YF. Antitumor activity and immunomodulatory effects of the intraperitoneal administration of Kanglaite in vivo in Lewis lung carcinoma. J Ethnopharmacol 2012; 143(2): 680-685.

38. Wu Y, Zhang J, Hong $Y$, Wang $X$. Effects of Kanglaite Injection on Serum miRNA-21 in Patients with Advanced Lung Cancer. Med Sci Monit 2018; 24: 2901-2906. 